\title{
Calidad de vida en adolescentes: Análisis desde las fortalezas personales y las emociones negativas
}

\section{Quality of life in adolescents: analysis from personal strengths and negative emotions}

\author{
Japcy Margarita Quiceno \\ Stefano Vinaccia \\ Universidad de San Buenaventura, Bogotá, Colombia
}

(Rec: 21 noviembre 2012 / Acept: 17 octubre 2014)

\begin{abstract}
Resumen
El objetivo de este estudio fue comparar las relaciones de las fortalezas personales y las emociones negativas sobre la calidad de vida de 686 adolescentes escolarizados de la ciudad de Bogotá, Colombia, según el sexo y el estrato socioeconómico. Instrumentos: escala de resiliencia adolescente ARS, escala de autotrascendencia adolescente STS, escala subjetiva de felicidad SHS, test de orientación de vida en jóvenes YLOT, inventario de depresión infantil CDI, inventario infantil de estresores cotidianos IIEC y The KIDSCREEN-52. Los resultados mostraron niveles moderados en calidad de vida y resiliencia, y niveles bajos de emociones negativas. El análisis de regresión lineal múltiple indicó que las fortalezas personales y las emociones negativas pesan sobre la calidad de vida más en los hombres y en el estrato socioeconómico medio y alto. Conclusión: la calidad de vida en adolescentes es mediada por la autotrascendencia, la felicidad y emociones negativas como depresión.

Palabras clave: calidad de vida, fortalezas personales, emociones negativas y adolescentes.
\end{abstract}

\begin{abstract}
The aim of this study was to compare the relationship of personal strengths and negative emotions on the quality of life of 686 adolescent students by sex and socioeconomic strata from Bogotá city, Colombia. Instruments: Adolescent Resilience Scale ARS, Self-Trascendence Scale, adolescent version STS, Subjective Happiness Scale SHS, The Youth Life Orientation Test YLOT, Children's Depression Inventory CDI, the Inventory of Daily Stressors Child IIEC and the KIDSCREEN-52. Results showed moderate levels in quality of life, resilience and low in negative emotions. It was found in the multiple linear regression analysis that personal strengths and negative emotions weigh on the quality of life in men more than women and in middle and high socioeconomic strata rather than low. Conclusion, quality of life in adolescents is mediated by self-transcendence and happiness and negative emotions as depression.

Keywords: quality of life, personal strengths, depression, stress, adolescents.
\end{abstract}

Correspondencia:

Japcy Margarita Quiceno, japcyps@hotmail.com, Universidad de San Buenaventura, Carrera 8H \# 172-08, Bogotá, Colombia. 


\section{Introducción}

Las investigaciones en el área de la psicología positiva, desde una perspectiva infanto-juvenil, son muy recientes y escasas, las cuales se han centrado en el estudio de las fortalezas personales como espiritualidad (trascendencia), optimismo, felicidad, gratitud, sentido de vida y sentido del humor, entre otros (Giménez, Vásquez y Hervás, 2010). Encontrándose en común en todos estos constructos que aminoran las emociones negativas como la depresión, el estrés y el pesimismo y aumentan las emociones positivas.

Al respecto, se ha encontrado en la literatura científica a nivel psicosocial, que las emociones positivas se asocian con la protección psicosocial (mayor conexión social y apoyo social percibido), el optimismo y un estilo de afrontamiento más adaptativo (Selligman, 2005). Todo esto reduce el riesgo de resultados adversos contra la salud física (Steptoe, Dockray y Wardle, 2009). Igualmente, las emociones positivas favorecen la madurez psicológica, aumentan la felicidad, la supervivencia (Carr, 2007; Fredrickson, 2004a; Fredrickson y Losada, 2005; Lyubomirsky, 2008), el bienestar (Hernangómez, Vásquez y Hervás, 2009), la autoestima (Góngora, 2008), los sentimientos de gratitud (Emmons y Shelton, 2002; Fredrickson, 2004b) y la autotrascendencia, ya que las emociones positivas que genera la espiritualidad fomentan la sensación de que la vida tiene sentido y ayudan a encontrar un significado positivo a los acontecimientos cotidianos y a los momentos de adversidad (Saroglou, Buxant y Tilquin, 2008). También se ha encontrado en revisiones de estudios una fuerte asociación de las emociones positivas con la resiliencia y el optimismo, especialmente cuando las personas se enfrentan a situaciones o sucesos duros o difíciles de la vida (Carrobles y Benavides-Pereira, 2009; Fernández-Abascal, 2009; Maganto y Maganto, 2010; Martín, 2009; Philippe, Lecours y Beaulieu-Pelletier, 2009), ya que los adolescentes que tienen las habilidades para ser resilientes tienen una menor probabilidad de deprimirse y una mayor probabilidad de mantener el bienestar emocional, la autoeficacia, el pensamiento optimista y servicial y el mantenimiento de una orientación de éxito y favorable calidad de vida (Noble y McGrath, 2005).

La calidad de vida infantil (CVI), según J.M. Quiceno, se define como "la percepción del bienestar físico, psicológico y social del niño(a) y adolescente dentro de un contexto cultural específico, de acuerdo a su desarrollo evolutivo y sus diferencias individuales". En esta área se cuenta con un paulatino aumento de investigaciones en los últimos años, ya sea para validación de pruebas o estudios que buscan analizar las relaciones de algunas patologías con este constructo, siendo éstos mayores en países anglosajones que en hispanoparlantes, y desde una posición más biomédica, encontrándose a nivel general una percepción más desfavorable de la calidad de vida relacionada con la salud (CVRS) de los niños y adolescentes que presentan alguna patología física, respecto a muestras de poblaciones de niños y adolescentes sanos. De otro lado, la CVRS se ve vulnerada por los estados emocionales negativos como la ansiedad y la depresión o los autoesquemas como la autoestima y la autoimagen (Quiceno y Vinaccia, 2013). Pero poco se sabe específicamente del impacto de variables positivas sobre las dimensiones de este constructo a nivel físico, psico-social y emocional en población adolescente.

Se hace necesario entonces, el desarrollo de estudios en la infancia/adolescencia en gran escala, desde una perspectiva de género y estratificación social, ya que los estudios no son aún concluyentes (Legood et al., 2009; Michel et al., 2009), especialmente en un país de regiones como Colombia, donde se menciona con frecuencia la palabra "calidad de vida" como necesidad en procesos de mejoramiento continuo en diferentes esferas sociales, en las políticas de salud pública, en la educación, etc., pero no se cuenta con investigaciones sólidas y concluyentes sobre el tema de la infancia y del peso de los factores de protección y de variables tanto positivas como negativas sobre la calidad de vida infantil. Es por eso que este estudio ex-post-facto correlacional tuvo como objetivo comparar las relaciones de las fortalezas personales (resiliencia, autotrascendencia, felicidad y optimismo) y las emociones negativas (pesimismo, depresión y estresores cotidianos) sobre la calidad de vida relacionada con la salud de 686 adolescentes escolarizados de la ciudad de Bogotá, Colombia, según el sexo y el estrato socioeconómico.

\section{Método}

\section{Participantes}

Participaron en el estudio 686 adolescentes hombres $(n=364,53.1 \%)$ y mujeres $(n=322,46.9 \%)$ entre los doce a dieciséis años, con una media de edad de trece años y ocho meses, pertenecientes a colegios privados de la ciudad de Bogotá de estrato bajo $(n=243,35.4 \%)$ y medio y alto $(n=443,64.6 \%)$,quienes cursaban los grados sexto a undécimo de básica secundaria, quienes fueron escogidos mediante muestreo no aleatorio de sujetos disponibles. Gran parte de la muestra tiene y vive con una familia de tipo nuclear $(n=387,56.4 \%)$ y en segundo orden vive en una 
familia donde la madre es jefe de familia $(n=111,16.2 \%)$. El resto convive con una familia extendida con padres $(n=79$, $11.5 \%)$, con padres y abuelos $(n=59,8.6 \%)$, con una familia compuesta $(n=42,6.1 \%)$ y con una familia extendida $\sin$ padres $(n=8,1.2 \%)$. El número de hermanos de la mayoría de los participantes fue entre uno $(n=311,45.3 \%)$ y dos $(n=171,24.9 \%)$, viéndose una tendencia también al único hijo ( $n=104,15.2 \%)$, mientras el resto de la muestra tiene entre tres a seis hermanos $(n=100,14.6 \%)$. A nivel general, los adolescentes no reportaron tener algún diagnóstico de enfermedad ( $n=577,84.1 \%)$, mientras que el 15.9\% $(n=109)$ reportó tener diagnóstico de enfermedades especialmente visuales y otorrinolaringológicas.

\section{Instrumentos}

Escala de resiliencia adolescente (Adolescent Resilience Scale, ARS): desarrollada por Oshio, Kaneko, Nagamine y Nakaya (2003) en Japón, traducida al idioma español por Restrepo (2009) y adaptada por Quiceno y Vinaccia (2010). La escala ARS consta de 21 ítems con un sistema de respuesta tipo Likert que oscila entre 1 ("definitivamente sí") a 5 ("definitivamente no") y comprende tres dimensiones (búsqueda de la novedad, regulación emocional y orientación positiva al futuro) y una escala total. A mayor puntuación, mayor resiliencia.

Escala de autotrascendencia versión adolescentes (Self-Trascendence Scale, adolescent version, STS): desarrollada originalmente por Reed (1987), se cuenta con la traducción y adaptación cultural colombiana (Quiceno y Vinaccia, 2010). La STS mide la autotrascendencia como un recurso psicosocial y espiritual importante en el desarrollo madurativo de las personas. Este cuestionario está conformado por 15 ítems y una sola dimensión con un sistema de respuesta tipo Likert que va de 1 a 4 puntos ( $1=$ nada; $4=$ mucho). Puntuaciones superiores en la escala STS indican altos niveles de autotrascendencia.

Escala subjetiva de felicidad (Subjective Happiness Scale, SHS): desarrollada originalmente por Lyubomirsky y Lepper (1999), presenta muy buen nivel de fiabilidad ( $\alpha$ $>$.80). Se cuenta con la adaptación cultural colombiana (Quiceno y Vinaccia, 2010). Esta escala consta de 4 ítems con un sistema de respuesta tipo Likert que va de 1 a 7 . A mayor puntuación, mayor percepción subjetiva de felicidad.

Test de orientación de vida en los jóvenes (The Youth Life Orientation Test, YLOT): desarrollada originalmente por Ey et al. (2005), se cuenta con la traducción y adaptación cultural colombiana (Quiceno y Vinaccia, 2010). Esta escala mide cómo ven el futuro los niños y adolescentes en cuanto son optimistas o pesimistas. Comprende 19 ítems con un sistema de respuesta tipo Likert que va de 0 (falso para mí) a 3 puntos (verdadero para mí). Contiene dos dimensiones, una de optimismo y otra de pesimismo. A mayor puntuación, mejor o peor optimismo.

Inventario de depresión Infantil (Children's Depression Inventory, CDI): diseñado originalmente por Kovacs (1992) en los Estados Unidosl, se cuenta con la versión en español de Davanzo et al. (2004). El coeficiente alfa de Cronbach encontrado fue de .85 . Consta de 27 ítems con un formato tipo Likert de 3 posibilidades de respuesta, 0 ausencia de síntomas, 1 síntoma moderado y 2 síntomas severos.

Inventario infantil de estresores cotidianos (IIEC): desarrollado originalmente por Trianes et al. (2009), presenta propiedades psicométricas adecuadas y se cuenta con la adaptación cultural colombiana (Quiceno y Vinaccia, 2010). "Este inventario evalúa exclusivamente pequeños fastidios, problemas y dificultades, que son considerados estresores cotidianos" (Trianes et al., 2009, p. 601), relacionados con la salud, escuela, pares y familia del adolescente. Consta de 22 ítems en un formato tipo Likert que va de 1 (nada estresante) a 5 puntos (muy estresante).

Cuestionario de salud y bienestar niños(as) y adolescentes (The KIDSCREEN-52): la versión original fue desarrollada por Herdman y el grupo europeo KIDSCREEN (2002), se cuenta con la adaptación colombiana (Colombia KIDSCREEN-52) realizada por Jaimes (2008). Este cuestionario comprende diez dimensiones y 52 ítems que se responden en una escala tipo Likert, con cinco alternativas de respuesta ("nunca" a "siempre") para las dimensiones: bienestar psicológico, estado de ánimo, autopercepción, autonomía, vida familiar, recursos económicos, amigos y apoyo social, entorno escolar y aceptación social. A su vez la dimensión bienestar físico tiene un sistema de respuesta tipo Likert con cinco alternativas que van de "excelente a "mala".

\section{Procedimiento}

Previo permiso de los representantes legales de los colegios que participaron en el estudio, se prosiguió con la captación de la muestra. Primero se informó a los padres de familia/acudientes sobre el propósito del estudio y luego se comunicó del estudio a los adolescentes cuyos padres autorizaron previamente su participación en la investigación. Tanto a los padres de familia/acudientes como a los adolescentes se les aclaró que podían retirarse del estudio en el momento que lo consideraran pertinente y que no recibirían 
ningún tipo de retribución económica. Los adolescentes que de manera libre y voluntaria decidieron participar fueron reunidos por grupos en las instalaciones de cada institución educativa, y se les entregaron los diferentes instrumentos psicométricos como la ficha de datos sociodemográficos para ser respondida de forma individual, contando con la ayuda del investigador a cargo en el momento que lo requirieran. El proceso de aplicación tuvo una duración de una hora.

\section{Resultados}

A continuación se presentan los análisis estadísticos del estudio. Para el análisis descriptivo de las variables edad, resiliencia, autotrascendencia, felicidad, optimismo/ pesimismo, depresión, estrés y calidad de vida infantil se utilizó la media como medida de tendencia central. Asimismo, se calculó la desviación típica y las puntuaciones mínimas y máximas de las diferentes dimensiones de los instrumentos psicométricos utilizados. Y respecto a las variables nominales (sexo, escolaridad, con quién vive el adolescente y tipo de enfermedad) y discretas (número de hermanos) se emplearon porcentajes. Se calculó además el coeficiente alfa de Cronbach para el análisis de fiabilidad de los instrumentos psicométricos. Se realizaron análisis de correlación de Pearson entre las variables que comprendió el estudio, clasificándolos según el sexo y el estrato socioeconómico. Por último, se empleó un modelo de regresión lineal múltiple para evaluar el peso de las variables predictoras como resiliencia, autotrascendencia, felicidad, optimismo/pesimismo, depresión y estrés sobre la calidad de vida infantil (variable criterio).

\section{Análisis descriptivo de las variables psicológicas del estudio}

En la tabla 1 se presentan los datos descriptivos de las variables psicológicas y el alfa de Cronbach de los instrumentos empleados, hallándose a nivel general de aceptable a muy buena fiabilidad en las diferentes dimensiones de los instrumentos psicométricos utilizados en el estudio.

Tabla 1. Análisis descriptivo de las variables psicológicas del estudio $(n=686)$.

\begin{tabular}{lccccc}
\hline & Media & $\begin{array}{c}\text { Desviación } \\
\text { Típica }\end{array}$ & Mínimo & Máximo & Alfa \\
\hline Resiliencia - ARS & 3.86 & 0.44 & 1 & 5 & 0.70 \\
Autotrascendencia - STS & 3.34 & 0.42 & 1 & 4 & 0.79 \\
Felicidad - SHS & 5.34 & 0.94 & 1 & 7 & 0.60 \\
Optimismo - YLOT & 14.33 & 3.00 & 1 & 19 & 0.65 \\
Pesimismo - YLOT & 4.11 & 3.54 & 0 & 18 & 0.71 \\
Depresión - CDI & 9.28 & 5.60 & 0 & 35 & 0.81 \\
Estresores cotidianos - IIEC & 2.19 & 0.51 & 1 & 4 & 0.78 \\
Calidad de vida - KIDSCREEN-52 & & & & & \\
Bienestar físico & 49.95 & 11.51 & 23 & 71 & 0.76 \\
Bienestar psicológico & 55.21 & 11.92 & 9 & 70 & 0.89 \\
Estado de ánimo & 42.93 & 12.55 & 7 & 71 & 0.87 \\
Autopercepción & 47.09 & 10.26 & 21 & 71 & 0.66 \\
Autonomía & 47.37 & 11.75 & 5 & 68 & 0.83 \\
Relación con padres y vida familiar & 51.93 & 13.60 & 12 & 69 & 0.90 \\
Recursos económicos & 48.90 & 10.33 & 24 & 65 & 0.90 \\
Amigos y apoyo social & 56.76 & 12.20 & 8 & 73 & 0.88 \\
Ambiente escolar & 50.10 & 10.83 & 17 & 73 & 0.83 \\
Aceptación social & 45.00 & 12.15 & 3 & 59 & 0.76 \\
\hline
\end{tabular}


En la tabla 1 se aprecia, en cuanto a la resiliencia, puntuaciones medias moderadas y en autotrascendencia y felicidad se observan puntuaciones medias altas. El optimismo tuvo puntuaciones medias más altas respecto al pesimismo, mientras que en depresión y estrés cotidiano los niveles medios son bajos. Por último, la calidad de vida tuvo puntuaciones medias moderadas en todas las dimensiones, menos en estado de ánimo y aceptación social, donde las puntuaciones son bajas.

\section{Análisis de correlación}

En la tabla 2 y 3 se presenta el análisis de correlación de Pearson entre las variables psicológicas que comprendió el estudio según el sexo y el estrato socioeconómico, hallándose correlaciones entre altas a bajas.

En la tabla 2 se observan asociaciones más fuertes en los hombres respecto a las mujeres en las fortalezas personales (resiliencia, autotrascendencia, felicidad y optimismo) con la calidad de vida. Por el contrario, en las emociones negativas (pesimismo, depresión y estresores cotidianos) hay mayor fuerza de correlación en las mujeres en comparación con los hombres con la calidad de vida. De igual manera, en la tabla 3 se observan asociaciones más fuertes en el estrato medio $\mathrm{y}$ alto respecto al estrato bajo en las fortalezas personales (resiliencia, autotrascendencia, felicidad y optimismo) y en las emociones negativas (pesimismo, depresión y estresores cotidianos) con la calidad de vida. Y por otro lado, se aprecian, tanto según el sexo como el estrato socioeconómico, relaciones entre las fortalezas personales y emociones negativas entre sí, y entre las fortalezas personales con las emociones negativas.

\section{Análisis de regresión lineal múltiple}

A continuación se presenta el análisis de regresión lineal múltiple de las fortalezas personales (resiliencia, autotrascendencia, felicidad y optimismo) y las emociones negativas (pesimismo, depresión y estresores cotidianos) sobre la CVRS según el sexo y el estrato socioeconómico.

\section{Análisis de regresión lineal múltiple entre las fortalezas personales y emociones negativas sobre la CVRS según el sexo}

En la tabla 4, en relación a los modelos finales de regresión lineal múltiple, se encontró en los hombres que la dimensión bienestar psicológico de CVRS alcanzó un coeficiente de determinación $R 2 c=49 \%$, explicado negativamente por la depresión y positivamente por las variables felicidad, autotrascendencia y resiliencia. Y en las mujeres igualmente se encontró un $R 2 c=49 \%$, explicado por las mismas variables que los hombres, a diferencia de la resiliencia siendo en éstas el pesimismo.

Por otro lado se aprecia en los hombres que la dimensión estado de ánimo de CVRS presentó un coeficiente de determinación $R 2 c=40 \%$, explicado negativamente por depresión, positivamente por resiliencia, negativamente por estresores cotidianos, positivamente por felicidad y negativamente por pesimismo. Mientras, en las mujeres el estado de ánimo de CVRS presentó un coeficiente de determinación $R 2 c=46 \%$, explicado negativamente por depresión y estresores cotidianos y positivamente por optimismo.

En cuanto a la relación con padres y vida familiar de CVRS, se observa un coeficiente de determinación $R 2 c=33 \%$, explicado positivamente por la autotrascendencia, negativamente por depresión, positivamente por felicidad y negativamente por estresores cotidianos. A su vez, en las mujeres hubo un coeficiente de determinación $R 2 c=40 \%$, explicado negativamente por la depresión, positivamente por autotrascendencia, negativamente por estresores cotidianos y positivamente por felicidad.

Respecto al ambiente escolar de CVRS, se encontró en los hombres un coeficiente de determinación $R 2 c=42 \%$, explicado negativamente por la depresión, positivamente por autotrascendencia y resiliencia, negativamente por estresores cotidianos y positivamente por felicidad. Mientras, en las mujeres el ambiente escolar tuvo un coeficiente de determinación $R 2 c=36 \%$, explicado negativamente por depresión, positivamente por autotrascendencia y negativamente por los estresores cotidianos.

Por otro lado, sólo en los hombres las siguientes variables de CVRS fueron representativas en los modelos finales de regresión lineal múltiple, encontrándose que la dimensión bienestar físico de CVRS tuvo un coeficiente de determinación $R 2 c=40 \%$, explicado negativamente por depresión, positivamente por felicidad, negativamente por estresores cotidianos y positivamente por autotrascendencia. De igual modo, la dimensión autonomía de CVRS presentó un coeficiente de determinación $R 2 c=33 \%$, explicado positivamente por optimismo, negativamente por depresión, positivamente por felicidad y autotrascendencia y negativamente por pesimismo. Por último, la dimensión amigos y apoyo social de CVRS alcanzó un coeficiente de determinación $R 2 c=34 \%$, explicado positivamente por autotrascendencia, negativamente por depresión y positivamente por felicidad y optimismo. 


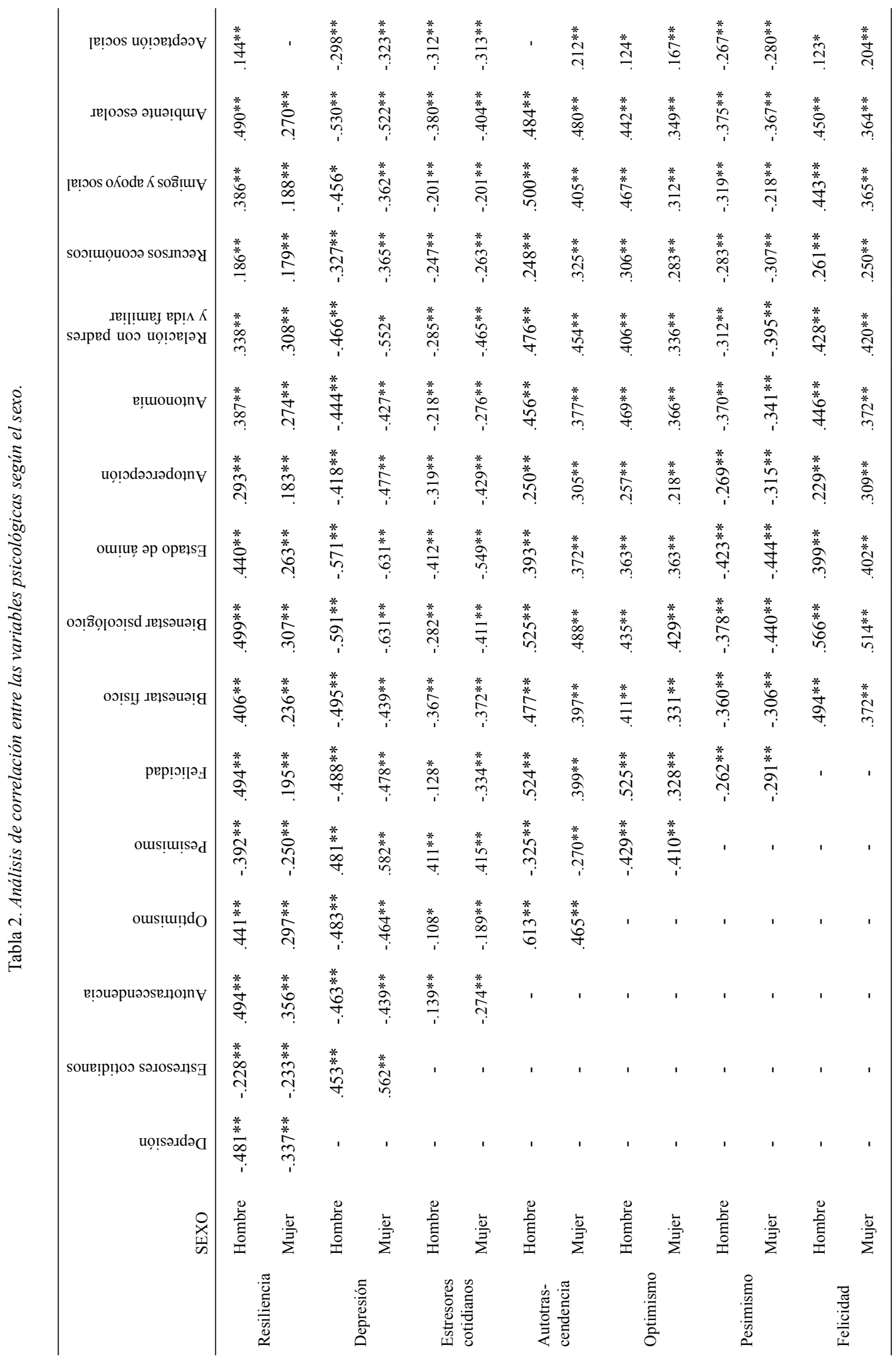




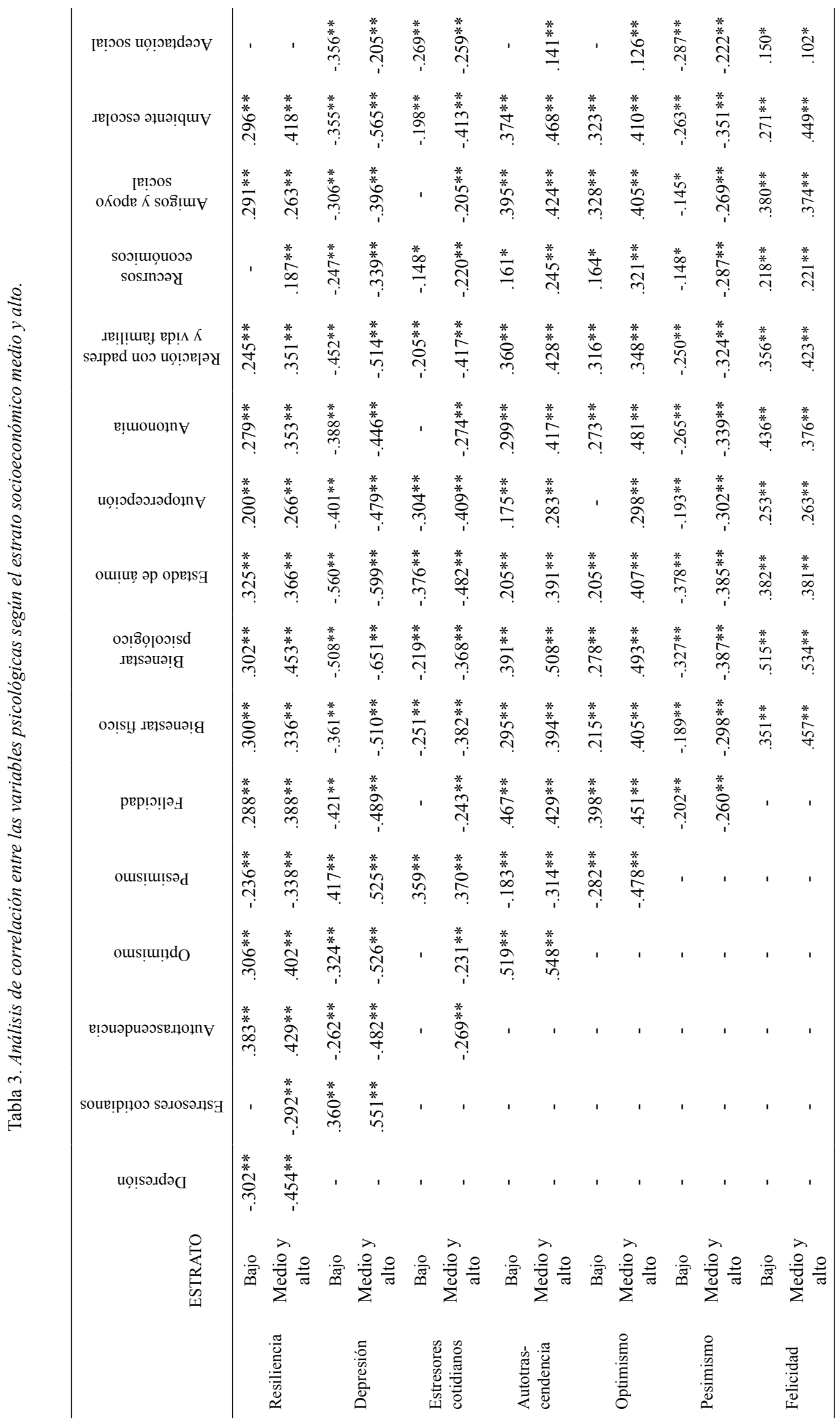


Tabla 4.

Análisis de las fortalezas personales y emociones negativas sobre la CVRS según el sexo.

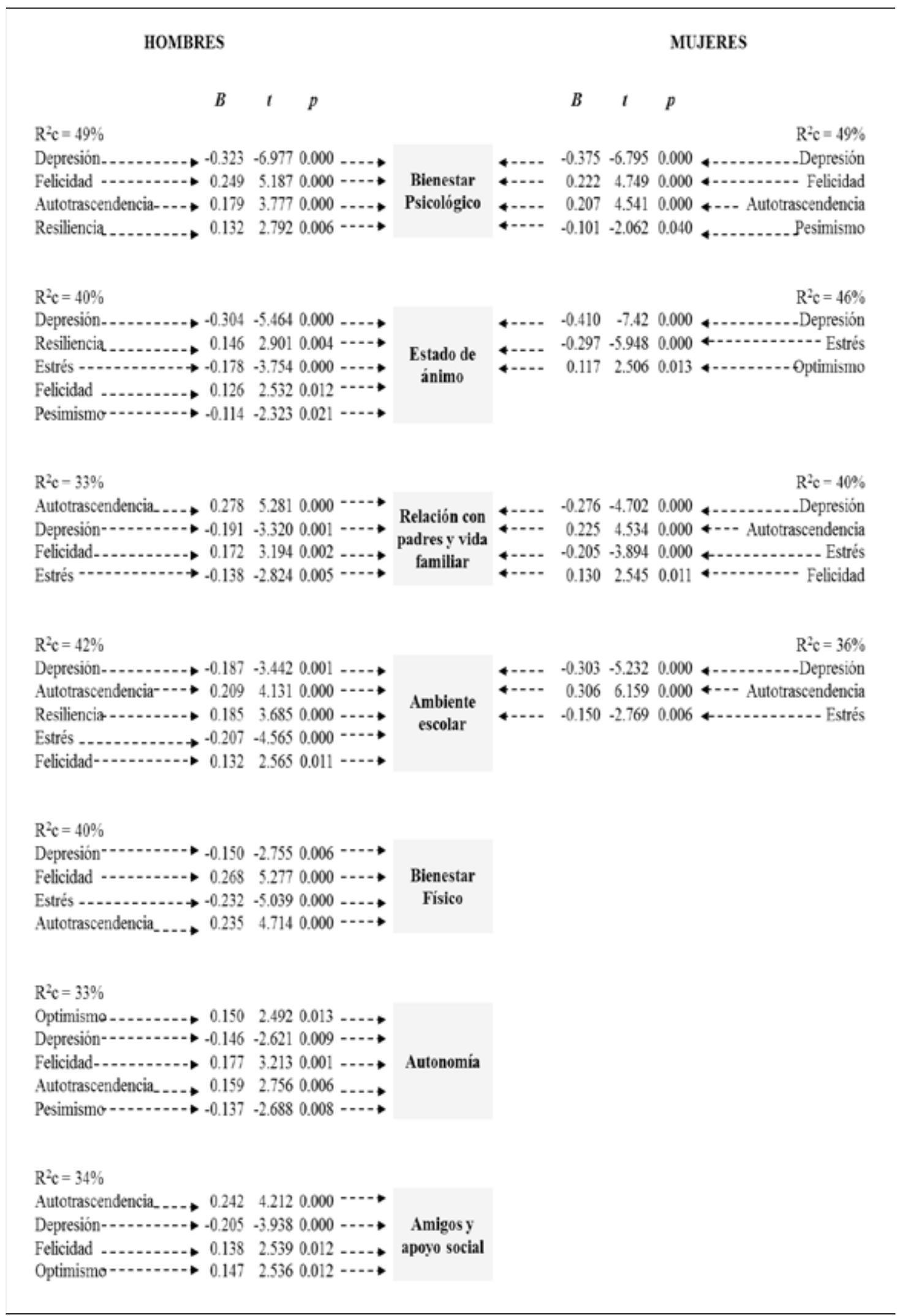


Tabla 5.

Análisis de las fortalezas personales y emociones negativas sobre la CVRS según el estrato.

ESTRATO MEDIO Y ALTO

$B \quad t \quad p$

$\mathrm{R}^{2} \mathrm{c}=52 \%$

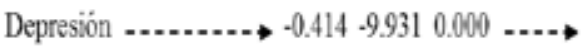

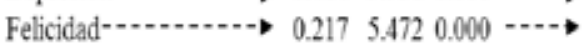

Autotrascendencia $-\rightarrow 0.1694 .2200 .000-\cdots$

Resiliencia 0.1092 .7810 .006
Bienestar

Psicológico



B $\quad t \quad p$
$\mathrm{R}^{2} \mathrm{c}=41 \%$

Depresión - - - - $0.362-6.8820 .000 \ldots$

Estrés _........ $0.232 .5 .2860 .000 \ldots$

Optimismo - - - $-\ldots .122 \quad 2.7200 .007 \ldots$

Felicidad-.......... $0.092 \quad 2.1180 .035 \ldots$

\section{ESTRATO BAJO}

$\mathrm{R}^{2} \mathrm{c}=34 \%$

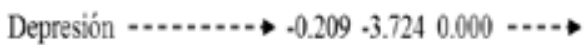

Felicidad -....... $0.228 \quad 4.9220 .000 \ldots$.

Estrés $\cdots \cdots \cdots$

Optimisme-... $0.1012 .0110 .045 \ldots$

Autotrascendencia $\ldots 0.0971 .9900 .047 \ldots$
Bienestar

Fisico

$\mathrm{R}^{2} \mathrm{c}=30 \%$

Optimismo - - $-\cdots 0.2564 .9460 .000-\cdots$

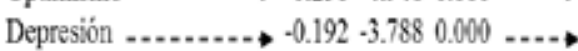

Autotrascendencia $\cdots 0.138 \quad 2.7570 .006 \quad \ldots$

Felicidad -........ $0.1072 .2370 .026 \ldots$

Autonomía

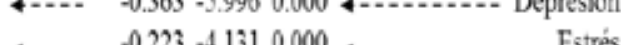

ánimo $\quad$-..- 0.1662 .9490 .004 -

4... 0.1542 .8670 .005 ......... Resiliencia

$\mathrm{R}^{2} \mathrm{c}=35 \%$

Depresión - - - $-0.2244 .1840 .000 \cdots$

Autotrascendencia $-\cdots 0.1884 .1550 .000 \ldots$

Estrés -

Felicidad -...... $0.1854 .0650 .000 \ldots \rightarrow$

Relación con

padres y vida

familiar

$\mathrm{R}^{2} \mathrm{c}=41 \%$

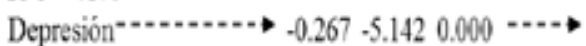

Autotrascendencia $\cdots 0.1824 .1150 .000 \cdots$

Felicidad $\cdots . .160 \quad 3.6450 .000 \quad \ldots$

Estrés $\cdots \cdots$

Ambiente

escolar 
Análisis de regresión lineal múltiple entre las fortalezas personales y emociones negativas sobre la CVRS según el estrato

En la tabla 5, en relación a los modelos finales de regresión lineal múltiple, se encontró en el estrato medio y alto que la dimensión bienestar psicológico de CVRS tiene un coeficiente de determinación $R 2 c=52 \%$, explicado negativamente por la depresión, y positivamente por las variables felicidad, autotrascendencia y resiliencia. Mientras que en el estrato bajo se encontró un coeficiente de determinación $R 2 c=39 \%$, explicado positivamente por felicidad, negativamente por depresión, positivamente por autotrascendencia y negativamente por pesimismo.

Por otro lado, se aprecia en el estrato medio y alto que la dimensión estado de ánimo de CVRS presentó un coeficiente de determinación $R 2 c=41 \%$, explicado negativamente por depresión y estresores cotidianos, y positivamente por optimismo y felicidad. Mientras que en el estrato bajo el estado de ánimo de CVRS alcanzó un coeficiente de determinación $R 2 c=39 \%$, explicado negativamente por depresión y estresores cotidianos y positivamente por felicidad y resiliencia.

Sólo en el estrato medio y alto las siguientes variables de CVRS fueron representativas en los modelos finales de regresión lineal múltiple, encontrándose que la dimensión bienestar físico de CVRS presentó un coeficiente de determinación $R 2 c=34 \%$, explicado negativamente por depresión, positivamente por felicidad, negativamente por estresores cotidianos y positivamente por optimismo y autotrascendencia. De igual modo, la dimensión autonomía de CVRS presentó un coeficiente de determinación $R 2 c=30 \%$, explicado positivamente por optimismo, negativamente por depresión, y positivamente por autotrascendencia y felicidad. Se halló además que la dimensión relación con padres y vida familiar de CVRS presentó un coeficiente de determinación $R 2 c=35 \%$, explicado negativamente por depresión, positivamente por autotrascendencia, negativamente por estresores cotidianos y positivamente por felicidad. Por último, la dimensión ambiente escolar de CVRS tuvo un coeficiente de determinación $R 2 c=41 \%$, explicado negativamente por depresión, positivamente por autotrascendencia y felicidad, negativamente por estresores cotidianos y positivamente por resiliencia.

\section{Discusión}

En el estudio participaron 686 adolescentes escolarizados de básica secundaria de ambos sexos pertenecientes a colegios privados de la ciudad de Bogotá, Colombia, de diferentes estratos socioeconómicos. Los colegios de estrato medio y alto estaban situados en el norte de la ciudad de Bogotá, con mensualidades entre 200 a 400 dólares americanos, mientras que los de estrato bajo estaban localizados en el sur de la ciudad y tenían una mensualidad de 30 dólares americanos. Es de anotar que la gran mayoría de los adolescentes vive con sus padres y hermanos o con la madre jefe de familia, aunque hay una tendencia a la familia con hijo único.

En relación a los instrumentos empleados en la investigación, es de aclarar que es la primera vez que se aplican en muestras colombianas de adolescentes escolarizados, con excepción de la escala KIDSCREEN-52 de calidad de vida, de la cual se cuenta con una adaptación cultural (Jaimes, 2008), la escala de resiliencia donde se tienen algunas caracterizaciones mas no validaciones con muestras de adolescentes escolarizados (Quiceno, Vinaccia y Grupo Fundación Flor de Liz, 2013; Restrepo, Vinaccia y Quiceno, 2011), igual que el inventario de depresión infantil CDI (Gómez, Alvis y Sepúlveda, 2003; Vinaccia et al., 2006). Es de anotar que todos los instrumentos tuvieron, en general, niveles de fiabilidad de aceptables a buenos.

En cuanto a la resiliencia los adolescentes mostraron una resiliencia moderada, es decir, tienen cierto interés hacia una amplia variedad de eventos y cierta compostura y control de sus emociones internas, pero sobre todo con un fuerte interés por tener metas hacia el futuro (Oshio et al., 2003). Los resultados de esta investigación son ligeramente superiores al estudio original de Oshio et al. (2003) en Japón (media=3.86 vs. 3.48), de Restrepo et al. (2011) en la ciudad de Medellín (media=3.86 vs. 3.57 ) y similar al de Quiceno, Vinaccia, Agudelo y González (2014) en un colegio privado de Bogotá (media $=3.86$ vs. 3.90 ).

En este estudio, la resiliencia tanto en hombres como en mujeres tuvo correlaciones con depresión de manera negativa $\mathrm{y}$, con autotrascendencia, optimismo, felicidad y algunas dimensiones de CVRS como bienestar físico, bienestar psicológico, estado de ánimo y ambiente escolar de manera positiva. En el análisis de regresión lineal múltiple, la resiliencia logra incidir sólo en la CVRS de los hombres a nivel de bienestar psicológico, estado de ánimo y ambiente escolar, pero en las mujeres la resiliencia no fue significativa sobre la CVRS. En cuanto a la resiliencia según el estrato socioeconómico bajo y, medio y alto, tuvo una correlación moderada y negativa con depresión y positiva con autotrascendencia, optimismo y las dimensiones de CVRS como bienestar psicológico y ambiente escolar. De acuerdo al análisis de regresión lineal múltiple, en el estrato socioeconómico medio y alto la resiliencia logra tener un peso significativo sobre la CVRS a nivel de bienestar 
psicológico y ambiente escolar, mientras que en el estrato bajo la resiliencia predice sobre el estado de ánimo. En tanto, la resiliencia tuvo menor peso sobre la CVRS en relación a las demás variables del estudio. Al respecto, Hjemdal, Vogel, Solem, Hagen y Stiles (2011) realizaron un estudio en Noruega con una muestra de 307 adolescentes escolarizados y después de controlar el sexo y la edad, hallaron que puntuaciones altas en resiliencia predecían puntuaciones bajas en emociones negativas como ansiedad, depresión, estrés y síntomas obsesivo-compulsivos. Igual que en este estudio se aprecia que independientemente del sexo y especialmente en el estrato bajo, la presencia de emociones negativas, como estrés, depresión, soledad, tristeza, incompetencia y resignación (estado de ánimo), en estos adolescentes tienen una relación inversamente proporcional con la resiliencia.

En cuanto a la espiritualidad en los jóvenes, los estudios se han desarrollado especialmente con adolescentes que tienen alguna patología crónica encontrándose un efecto positivo en el bienestar psicológico (Hendricks-Ferguson, 2006) y en muestras de adolescentes sin patologías crónicas se ha hallado que la espiritualidad tiene una cualidad positiva, ayuda a hacer frente a situaciones difíciles y promueve la transformación del comportamiento cívico y conductas pro sociales (Roehlkepartain, Benson, Ebstyne-King y Wagener, 2005). La enfermera norteamericana Pamela G. Reed desarrolló la teoría de la autotrascendencia definiéndola como una capacidad evolutiva y un recurso potencial en el desarrollo madurativo de las personas de naturaleza no sólo espiritual sino psicosocial, donde la persona va "más allá de sus puntos de vista inmediatos y restringidos sobre sí mismos y el mundo", para conseguir la sensación continua de plenitud (Coward, 2007; Reed, 2003). Respecto a los estudios de autotrascendencia, basados en el modelo de Pamela G. Reed con muestras de adolescentes y adultos jóvenes son igualmente pocos (comunicación personal, 7 de agosto, 2012), encontrándose en puntuaciones medias muy similares al de esta investigación (media $=3.43$ vs. 3.30 , 3.31 y 3.37), indicando que los adolescentes de este estudio son bastante autotrascendentes.

De igual modo, en este estudio la autotrascendencia tanto en hombres como en mujeres tuvo correlaciones positivas con optimismo, felicidad y, con las dimensiones de CVRS como bienestar físico, bienestar psicológico, autonomía, relación con padres y vida familiar, amigos y apoyo social y, ambiente escolar. En el análisis de regresión lineal múltiple en los hombres, la autotrascendencia tuvo un peso significativo sobre las dimensiones de CVRS como bienestar físico, bienestar psicológico, autonomía, relación con padres y vida familiar, amigos y apoyo social $\mathrm{y}$, ambiente escolar, mientras que en las mujeres sólo la autotrascendencia incide sobre el bienestar psicológico, la relación con padres y vida familiar y, ambiente escolar. En cuanto a la autotrascendencia según el estrato socioeconómico bajo y, medio y alto, tuvo correlaciones positivas con las dimensiones de CVRS como bienestar psicológico, autonomía, relación con padres y vida familiar, amigos y apoyo social y, ambiente escolar. De acuerdo al análisis de regresión lineal múltiple, en el estrato socioeconómico bajo y, medio y alto, la autotrascendencia predice sobre la dimensión bienestar psicológico de CVRS y además sólo en el estrato medio y alto la autotrascendencia tuvo un peso significativo sobre el bienestar físico, autonomía, relación con padres y vida familiar y, ambiente escolar. Al respecto, los resultados de este estudio son similares al de Quiceno et al. (2014), donde igualmente hubo correlaciones entre autotrascendencia con las dimensiones bienestar físico, bienestar psicológico y relación con padres y vida familiar del KIDSCREEN-52.

Por otro lado, los adolescentes de este estudio se perciben felices. Según Lyubomirsky (2008), la felicidad es "la experiencia de alegría, satisfacción o bienestar positivo, combinada con la sensación de que nuestra vida es buena, tiene sentido y vale la pena" (p. 48). Los resultados de esta investigación son superiores a los datos de Vera-Villarroel, Celis-Atenas y Córdova-Rubio (2011) en un estudio con adolescentes chilenos (media $=5.34$ vs. 4.90 ) y ligeramente inferiores al estudio de Quiceno et al. (2014) (media $=5.34$ vs. 5.88).

La felicidad, tanto en hombres como en mujeres, tuvo correlaciones positivas con las dimensiones de CVRS como bienestar físico, bienestar psicológico, estado de ánimo, autonomía, relación con padres y vida familiar, amigos y apoyo social y, ambiente escolar. En el análisis de regresión lineal múltiple, la felicidad incide en los hombres sobre todas las dimensiones de CVRS como bienestar físico, bienestar psicológico, estado de ánimo, autonomía, amigos y apoyo social, relación con padres y vida familiar y, ambiente escolar, mientras que en las mujeres sólo la felicidad tiene un peso significativo sobre el bienestar psicológico y la relación con padres y vida familiar. En cuanto a la felicidad según el estrato socioeconómico bajo $\mathrm{y}$, medio y alto, tuvo asociaciones positivas con las dimensiones de CVRS como bienestar físico, bienestar psicológico, autonomía, relación con padres y vida familiar y, ambiente escolar. De acuerdo al análisis de regresión lineal múltiple, en el estrato socioeconómico bajo y, medio y alto, la felicidad incide sobre las dimensiones de CVRS como bienestar 
psicológico y estado de ánimo, mientras que en el estrato medio y alto sólo la felicidad tiene un peso significativo sobre el bienestar físico, autonomía, relación con padres y vida familiar y, ambiente escolar. Los datos de este estudio son afines a los de Quiceno et al. (2013), donde igualmente la felicidad correlacionó y tuvo una incidencia positiva sobre las dimensiones bienestar psicológico y relación con padres y vida familiar del KIDSCREEN-52.

En cuanto a la orientación de vida, según Ey et al. (2005) es un constructo que hace relación a cómo el niño ve o percibe su futuro, si de manera optimista o pesimista. El optimismo está ligado a las cosas buenas, es decir mejores amistades, mejor autoestima y mejor comportamiento. Por el contrario, el pesimismo es la preocupación por cosas negativas y sentimientos de tristeza, lo que lleva a un riesgo de depresión y problemas de comportamiento. Al respecto, los resultados de esta investigación son similares a las puntuaciones del estudio original de Ey et al. (2005) con adolescentes norteamericanos en optimismo (media= 14.33 vs 14.4 ) y difieren en pesimismo (media $=4.11$ vs 5.9), percibiéndose que los adolescentes de este estudio son poco pesimistas.

Los estudios sobre optimismo en adolescentes han sido de tipo descriptivo correlacional (Weber, Puskar y Ren, 2010), pero poco se conoce sobre estudios correlacionales que analicen las diferencias del optimismo/pesimismo según las características sociodemográficas en jóvenes. En cuanto a la variable optimismo, tanto en hombres como en mujeres tuvo correlaciones positivas con felicidad y con las dimensiones de CVRS como bienestar físico, bienestar psicológico, autonomía, relación con padres y vida familiar, amigos y apoyo social y ambiente escolar, y negativas con la variable pesimismo. En el análisis de regresión lineal múltiple, el optimismo incide sobre la CVRS de los hombres a nivel de la autonomía y, amigos y apoyo social, y en las mujeres sólo el optimismo incide en el estado de ánimo de la CVRS. En cuanto al optimismo según el estrato socioeconómico bajo y, medio y alto, se encontró una correlación negativa con pesimismo y positiva con felicidad y con las dimensiones de CVRS como bienestar físico, bienestar psicológico, estado de ánimo, autonomía, amigos y apoyo social y, ambiente escolar. De acuerdo al análisis de regresión lineal múltiple en el estrato socioeconómico medio y alto, el optimismo logra incidir sobre la CVRS a nivel del estado de ánimo, bienestar físico y autonomía, y esta variable en el estrato bajo incide sobre el bienestar psicológico de la CVRS. De acuerdo al pesimismo según el sexo, tanto en hombres como en mujeres tuvo correlaciones negativas con las dimensiones bienestar psicológico y estado de ánimo de CVRS. En el análisis de regresión lineal múltiple, el pesimismo tiene un peso significativo sobre el estado de ánimo de CVRS en los hombres, mientras que en las mujeres el pesimismo tiene un peso significativo sobre el bienestar psicológico de CVRS. En cuanto al pesimismo según el estrato socioeconómico bajo y, medio y alto, se encontró correlaciones bajas y negativas con felicidad y todas las dimensiones de CVRS. De acuerdo al análisis de regresión lineal múltiple en el estrato socioeconómico bajo, el pesimismo incide solamente sobre el bienestar psicológico de CVRS. Al respecto, Piko, Luszczynska y Fitzpatrick (2013) en Hungría, con una muestra de 881 adolescentes, hallaron que el estatus socioeconómico puede generar desigualdades sociales en la salud percibida y la depresión de los adolescentes a través del apoyo social de los padres, especialmente de la madre, desigualdades que pueden estar explicadas por las diferencias de recursos a nivel material, emocional y de seguridad brindados por los padres. Por tanto, los padres que ofrecen a sus hijos apoyo social, independientemente del nivel socioeconómico, pueden fortalecer o debilitar el optimismo durante los procesos de socialización. Como se aprecia en este estudio que, independientemente del sexo, el optimismo se vincula positivamente con la relación con padres y vida familiar de CVRS.

En cuanto a la variable depresión infantil que "es un desorden caracterizado por una alteración en el estado de ánimo, acompañada de cambios de comportamiento a nivel escolar, familiar y social" (Del Barrio, 2000, p. 23), no se encontró en esta investigación niveles clínicamente significativos (media $=9.28$ ) según los puntos de corte de 17 puntos por Craighead, Curry e Ilardi (1995) y de 19 puntos de la autora del inventario de depresión infantil de Kovacs (1992), lo que es afín a otros estudios colombianos como el de Vinaccia et al. (2006) con estudiantes del oriente antioqueño (media=10.52), el de Gómez et al. (2003) con estudiantes de Medellín (media=7.94) y el de Restrepo et al. (2011) con estudiantes de un colegio público de Medellín (media $=9.39$ ).

En cuanto a la depresión según sexo y estrato socioeconómico, ésta fue la variable que tuvo mayor fuerza de correlación con todas las variables del estudio y un peso negativo sobre todas las dimensiones de la CVRS. La depresión fue más significativa en las féminas, encontrándose correlaciones positivas con estresores cotidianos y pesimismo y, negativas con autotrascendencia, optimismo y felicidad y con las dimensiones de CVRS como bienestar físico, bienestar psicológico, estado de ánimo, autopercepción, autonomía, relación con padres y vida familiar, amigos y apoyo social y, ambiente escolar. En el análisis de regresión lineal múltiple, 
la variable depresión incide sobre las dimensiones de CVRS como bienestar psicológico, estado de ánimo, relación con padres y vida familiar $y$, ambiente escolar tanto en hombres como en mujeres. Por otro lado, esta variable sólo en los hombres tuvo un peso significativo sobre las demás dimensiones de CVRS como bienestar físico, autonomía y, amigos y apoyo social. En cuanto a la depresión según el estrato socioeconómico bajo y, medio y alto se encontró asociaciones positivas con estresores cotidianos y pesimismo, y negativas con autotrascendencia, optimismo, felicidad y las dimensiones de CVRS como bienestar físico, bienestar psicológico, estado de ánimo, autonomía, autopercepción, relación con padres y vida familiar y, ambiente escolar. De acuerdo al análisis de regresión lineal múltiple, en el estrato socioeconómico bajo y, medio y alto, la variable depresión incide sobre las dimensiones de CVRS como bienestar psicológico y estado de ánimo, mientras que en el estrato medio y alto la depresión tuvo un peso significativo en las demás dimensiones de CVRS como bienestar físico, autonomía, relación con padres y vida familiar y, ambiente escolar. Restrepo et al. (2011) hallaron en su investigación con adolescentes colombianos correlaciones negativas entre depresión medida con el cuestionario CDI con la calidad de vida a nivel de bienestar emocional, familia y escuela medido con el cuestionario Kid-Kindl y la depresión tuvo una influencia negativa sobre toda la calidad de vida del Kid-Kindl. Igual que en este estudio, independientemente del sexo y el estrato socioeconómico, la depresión fue la variable que tuvo un peso negativo importante en el estado de ánimo, la relación con padres y el ambiente escolar de la CVRS. Por otro lado, Weber et al. (2010) desarrollaron un estudio en Estados Unidos con adolescentes escolarizados y hallaron que los síntomas depresivos tuvieron una relación inversamente proporcional con el apoyo de amigos, familiar y social, la autoestima y el optimismo. Lo que es afín con este estudio donde amigos y apoyo social independientemente del sexo se asoció negativamente con depresión, pero a nivel del análisis de regresión lineal, la depresión incide sólo en los hombres. De otro lado, la depresión, independientemente del sexo y el estrato, incide y se vincula con la relación con padres y vida familiar, e independientemente del sexo y el estrato la depresión se asocia negativamente con autopercepción (autoestima) y el optimismo de la CVRS.

En cuanto a los estresores cotidianos, "son las frustrantes demandas y contrariedades que acarrea la interacción cotidiana con el medio ambiente... Se trata de sucesos o circunstancias de alta frecuencia, baja intensidad y alta predictibilidad" (Trianes et al., 2009, p. 598). Además, Trianes et al. (2009) plantean, por un lado, que los niños ante un mismo estresor pueden tener experiencias distintas, y por otro, que el estrés diario puede afectar de manera importante el área emocional, a diferencia del estrés originado por eventos vitales estresantes o permanentes.Al respecto, en un estudio realizado por Escobar, Fernández-Baena, Miranda, Trianes y Cowie (2011) se encontró niveles bajos de estrés diario, al igual que en este estudio, donde los adolescentes reportaron tener poco estrés a nivel de estresores como la salud, la familia y la escuela.

Respecto a la variable estresores cotidianos, tuvo correlaciones más fuertes y negativas en mujeres en las dimensiones de CVRS como bienestar psicológico, estado de ánimo, autopercepción, relación con padres y vida familiar y, ambiente escolar, y con pesimismo donde la relación fue negativa. En el análisis de regresión lineal múltiple, los estresores cotidianos tanto en hombres como en mujeres predicen la CVRS a nivel de estado de ánimo, relación con padres y vida familiar y ambiente escolar, y por otro lado, sólo en los hombres los estresores cotidianos inciden sobre el bienestar físico de CVRS. En cuanto al estrato socioeconómico bajo $\mathrm{y}$, medio y alto, los estresores cotidianos se asociaron negativamente con las dimensiones de CVRS como estado de ánimo, autopercepción, relación con padres y vida familiar y, ambiente escolar. De acuerdo al análisis de regresión lineal múltiple en el estrato socioeconómico bajo y, medio y alto, los estresores cotidianos predicen la CVRS a nivel de estado de ánimo, mientras que esta variable en el estrato medio y alto influyen sobre el bienestar físico, relación con padres y vida familiar $\mathrm{y}$, ambiente escolar de la CVRS. De igual modo, según el sexo y el estrato socioeconómico se encontraron relaciones negativas bajas entre estrés cotidiano con aceptación social (bullying). Trianes et al. (2009) plantean que en otras investigaciones se han reportado como factores de estrés infantil que amenazan la salud y el desarrollo del adolescente, la preocupación por la imagen corporal, los problemas académicos y el bullying, problemas con los iguales y conflictos de relación en la familia, lo que es afín a lo encontrado en este estudio donde la autopercepción (imagen corporal) y la aceptación social (bullying), independientemente del sexo y el estrato, tuvo asociaciones negativas con estresores cotidianos. Asimismo, independientemente del sexo, los estresores cotidianos se vinculan e inciden sobre la relación con padres y vida familiar de CVRS, y los estresores cotidianos, tanto en el estrato bajo $\mathrm{y}$, medio y alto, se asocian negativamente con el ambiente escolar de CVRS, pero más específicamente a nivel de regresión lineal múltiple los estresores cotidianos 
inciden en el ambiente escolar de la CVRS de los y las adolescentes de estrato medio y alto.

A nivel general, los adolescentes de este estudio tienen una percepción adecuada dentro de los límites normales de acuerdo a la etapa de desarrollo evolutivo de su calidad de vida relacionada con la salud, aunque es de resaltar que las dimensiones bienestar psicológico y amigos y apoyo social tienen los niveles medios más altos en el cuestionario, mientras que el estado de ánimo y la aceptación social (bullying) ameritan atención clínica. Estos resultados son similares a los de Quiceno et al. (2013) con adolescentes de colegios públicos de Bogotá.

En conclusión, se aprecia en este estudio que las fortalezas personales (resiliencia, autotrascendencia, felicidad y optimismo) y las emociones negativas (pesimismo, depresión y estresores cotidianos), tanto en los y las adolescentes, logran tener un peso sobre la CVRS a nivel de bienestar psicológico, estado de ánimo, relación con padres y vida familiar y, ambiente escolar, mientras que en los hombres las demás dimensiones de CVRS como bienestar físico, autonomía y, amigos y apoyo social se ven influidas por las fortalezas personales y las emociones negativas. En cuanto al estrato socioecómico, ya sea bajo o medio y alto, las fortalezas personales y las emociones negativas tienen un peso significativo sobre el bienestar psicológico y estado de ánimo de la CVRS, y sólo en el estrato socioeconómico medio y alto las fortalezas personales y las emociones negativas logran predecir sobre las demás dimensiones de CVRS como bienestar físico, autonomía, relación con padres y vida familiar $\mathrm{y}$, ambiente escolar.

En definitiva, más en los hombres que en las mujeres y más en el estrato socioeconómico medio y alto que en el bajo, las fortalezas personales y las emociones negativas logran tener impacto sobre la calidad de vida infantil. Los resultados llevan entonces a sugerir el desarrollo de programas de intervención focalizados en las fortalezas personales de los adolescentes considerando las diferencias por estrato socioeconómico y el género, con el fin de mejorar su calidad de vida relacionada con la salud en esta etapa de desarrollo.

\section{Referencias}

Carr, A. (2007). Psicología positiva: la ciencia de la felicidad. Barcelona, España: Paidós.

Carrobles, J. A. y Benavides-Pereira, A. M. (2009). El estrés y la psicología positiva. En E. G. Fernández-Abascal (Eds.), Emociones Positivas (pp. 363-373). Madrid, España: Editorial Pirámide.

Coward, D.D. (2007). Teoría de la autotrascendencia. En A. Marriner y M. Raile (Eds.), Modelos y teorías en enfermería (pp. 647-667). Madrid, España: Elsevier.
Craighead, W.E., Curry, J.C. yllardi, S.S. (1995). Relationship of Children $>$ Depression Inventory factors to major depression among adolescents. Psychological Assessment, 7(1), 171-176.

Davanzo, P., Kerwin, L., Nikore, V., Esparza, C., Forness, S. y Murelle, L. (2004). Spanish translation and reability testing of the child depression inventory. Child Psychiatry and Humane Development, 35, 75-92.

Del Barrio, M. V. (2000). La depresión infantil: factores de riesgo y posibles soluciones. Málaga, España: Aljibe.

Emmons, R. A. y Shelton, C. M. (2002). Gratitude and the science of positive psychology. In C. R. Snyder y J. López (Eds.), Handbook of positive psychology (pp. 459-471). Oxford University Press.

Escobar, M., Fernández-Baena, F. J., Miranda, J., Trianes, M. V. y Cowie, H. (2011). Low peer acceptance and emotional/behavioural maladjustment in schoolchildren: Effects of daily stress, coping and sex. Anales de Psicología, 27(2), 412-417.

Ey, S., Hadley, W., Allen, D. N., Palmer, S., Klosky, J., Deptula, D., et al. (2005). A new measure of children's optimism and pessimism: the youth life orientation test. Journal of Child Psychology and Psychiatry, 46(5), 548-558.

Fernández-Abascal, E. G. (2009). Emociones Positivas, Psicología Positiva y Bienestar. En E. G. Fernández-Abascal (Eds.), Emociones Positivas (pp. 27-47). Madrid, España: Editorial Pirámide.

Fredrickson, B. L. (2004a). El poder de los buenos sentimientos. Mente $y$ Cerebro, 8, 74-78.

Fredrickson, B. L. (2004b). Gratitude, like other positive emotions, broadens and builds. In Emmons, R. A. y M. E. McCullough (Eds.), The Psychology of Gratitude (pp. 145-166). New York: Oxford University Press.

Fredrickson, B. L. y Losada, M. F. (2005). Positive affect and the complex dynamics of human flourishing. American Psychologist, 60(7), 678-686.

Giménez, M., Vásquez, C. \& Hervás, G. (2010). El análisis de las fortalezas psicológicas en la adolescencia: Más allá de los modelos de vulnerabilidad. Psychology, Society \& Education, 2(2), 97-116.

Gómez, I., Alvis, A. y Sepúlveda, M. F. (2003). Características psicométricas del instrumento. Children Depression Inventory (CDI) para detectar sintomatología depresiva en niños de 8 a 12 años de la ciudad de Medellín. En PSICOG (Eds.), Investigaciones de psicología clínica-cognitiva comportamental en la ciudad de Medellín. Medellín, Colombia: U de A.

Góngora, V. C. (2008). La autoestima. En M. M. Casullo (Ed.), Prácticas en Psicología Positiva (pp. 81-110). Buenos Aires: Lugar.

Hendricks-Ferguson, V. (2006). Relationships of age and gender to hope and spiritual well-being among adolescents with cancer. Journal of Pediatric Oncology Nursing, 23(4), 189-199.

Herdman, M., Rajmil, L., Ravens-Sieberer, U., Bullinger, M., Power, M., Alonso, J. y the European KIDSCREEN and DISABKIDS groups (2002). Expert consensus in the development of a European healthrelated quality of life measure for children and adolescents: a Delphi study. Acta Pediátrica, 91(12), 1385-1390.

Hernangómez, L., Vásquez, C. y Hervás, G. (2009). El paisaje emocional a lo largo de la vida. En C. Vázquez y G. Hervás (Eds.), La ciencia del bienestar: fundamentos de psicología positiva (pp. 143-179). Madrid, España: Alianza Editorial.

Hjemdal, O., Vogel, P. A., Solem, S., Hagen, K. y Stiles, T. C. (2011). The relationship between resilience and levels of anxiety, depression, and obsessive-compulsive symptoms in adolescents. Clinical Psychology \& Psychotherapy, 18(4), 314-321.

Jaimes, M. L. (2008). Adaptación cultural a la población escolar colombiana del KIDSCREEN y del VSP-A, instrumentos de calidad de vida relacionada con la salud (CVRS) para niños(as) y adolescentes (Tesis doctoral inédita). Universidad de Alicante, Alicante.

Kovacs, M. (1992). Children Depresión Inventory CDI (manual). Toronto: Multihealth Systems.

Legood, R., Coen, P. G., Knox, K., Viner, R.M., El Bashir, H. y Christie, D. (2009). Health related quality of life in survivors of pneumococcal meningitis. Acta Pediátrica, 98(3), 543-547.

Lyubomirsky, S. (2008). La ciencia de la felicidad: Un método comprobado para conseguir el bienestar. Barcelona, España: Ediciones Urano. 
Lyubomirsky, S. y Lepper, H. S. (1999). A Measure of Subjective Happiness: preliminary reliability and construct validation. Social Indicators Research, 46(2), 137-155.

Maganto, C. y Maganto, J. M. (2010). Cómo potenciar las emociones positivas y afrontar las negativas. Madrid, España: Pirámide.

Martín, M. D. (2009). Emociones Positivas y Salud. En E. G. FernándezAbascal (Eds.), Emociones Positivas (pp. 393-420). Madrid, España: Editorial Pirámide.

Michel, G., Bisegger, C., Fuhr, D. C., Abel, T. y KIDSCREEN group (2009). Age and gender differences in health-related quality of life of children and adolescents in Europe: a multilevel analysis. Quality of Life Research, 18(9), 1147-1157.

Noble, T. y McGrath, H. (2005). Emotional growth-helping children and families "bounce back". Australian Family Physician, 34(9), 749-752.

Oshio, A., Kaneko, H., Nagamine, S. y Nakaya, M. (2003). Construct validity of the Adolescent Resilience Scale. Psychological Reports, 93, 1217-1222.

Philippe, F. L., Lecours, S. y Beaulieu-Pelletier, G. (2009). Resilience and Positive Emotions: examining the role of emotional memories. Journal of Personality, 77(1), 139-176.

Piko, B. F., Luszczynska, A. y Fitzpatrick, K. M. (2013). Social inequalities in adolescent depression: The role of parental social support and optimism. The International Journal of Social Psychiatry,59(5), 474-481.

Quiceno, J. M. y Vinaccia, S. (2010). Psicología Positiva e infancia: análisis exploratorio de instrumentos. Manuscrito no publicado, Universidad Autónoma de Madrid, España.

Quiceno, J. M. y Vinaccia, S. (2013). Calidad de vida relacionada con la salud infantil: una aproximación desde la enfermedad crónica. Psychologia: Avances de la Disciplina, 7(2), 69-86.

Quiceno, J. M., Vinaccia, S., Agudelo, L. Z. y González, D. C. (2014). Factores salutogénicos y patogénicos: una exploración desde la calidad de vida infantil. Revista Argentina de Clínica Psicológica, 23(1), 81-93.

Quiceno, J. M., Vinaccia, S. y Grupo Fundación Flor de Liz (2013). Calidad de vida, factores salutogénicos e ideación suicida en adolescentes. Revista Terapia Psicológica, 31(2), 263-271.

Reed, P. G. (1987). Constructing a conceptual framework for psychosocial nursing. Journal of Psychosocial Nursing, 25(2), 24-28.

Reed, P. G. (2003). The theory of Self-Transcendence. In M. J. Smith y P. Liehr (Eds.), Middle range theories in nursing New York: Springer.

Restrepo, C. (2009). Anteproyecto de investigación: Evaluación de la calidad de vida, la resiliencia y la depresión en adolescentes escolarizados de la ciudad de Medellín (Manuscrito no publicado). Universidad de San Buenaventura, Medellín, Colombia.
Restrepo, C., Vinaccia, S. y Quiceno, J. M. (2011). Resiliencia y Depresión: Un estudio exploratorio desde la calidad de vida en la adolescencia. Suma Psicológica, 18(2), 41-48.

Roehlkepartain, E. C., Benson, P. L., Ebstyne-King, P. y Wagener, L. (2005). The Handbook of Spiritual Development in Childhood and Adolescence. In E. C. Roehlkepartain, P. L. Benson, P. Ebstyne-King y L. Wagener (Eds.), Spiritual development in childhood and adolescence: moving to the scientific mainstream. EEUU: SAGE Publications.

Saroglou, V., Buxant, C. y Tilquin, J. (2008). Positive emotions as leading to religion and spirituality.The Journal of Positive Psychology, 3(3), 165-173.

Selligman, M. (2005). La auténtica felicidad. Barcelona, España: Byblos. Steptoe, A., Dockray, S. y Wardle, J. (2009). Positive Affect and Psychobiological Processes Relevant to Health. Journal of Personality, 77(6), 1747-1776

Trianes, M. V., Blanca, M. J., Fernández, F. J., Escobar, M., Maldonado, E. F. y Muñoz, A. M. (2009). Evaluación del estrés infantil: Inventario Infantil de Estresores Cotidianos (IIEC). Psicothema, 21(4), 598-603.

Vera-Villarroel, P., Celis-Atenas, K. y Córdova-Rubio, N. (2011). Evaluación de la Felicidad: Análisis Psicométrico de la Escala de Felicidad Subjetiva en Población Chilena. Terapia Psicológica, 29(1), 127-133.

Vinaccia, S., Gaviria, A. M., Atehortúa, L. F., Martínez, P. H., Trujillo, C. y Quiceno, J. M. (2006). Prevalencia de depresión en niños escolarizados entre 8 y 12 años del oriente antioqueño, a partir del "Child Depression Inventory" -CDI-. DIVERSITAS: Perspectivas en Psicología, 2(2), 217-227.

Weber, S., Puskar, K. R. y Ren, D. (2010). Relationships between depressive symptoms and perceived social support, self-esteem \& optimism in a sample of rural adolescents. Issues in Mental Health Nursing, 31(9), 584-588. 
